\title{
Estrategias de control de calidad de energía en microrredes rurales
}

\section{Control strategies for power quality in rural microgrids}

\author{
Nelson González ${ }^{1}$, Carlos Cusgüen ${ }^{2}$, Eduardo Mojica-Nava ${ }^{3}$, Andrés Pavas ${ }^{4}$ \\ ${ }^{1}$ Programa de investigación sobre adquisición y análisis de señales PAAS-UN, Facultad de Ingeniería, Universidad Nacional- \\ Bogotá, Colombia. Email: nygonzalezc@unal.edu.co \\ ${ }^{2}$ Programa de investigación sobre adquisición y análisis de señales PAAS-UN, Facultad de Ingeniería, Universidad Nacional- \\ Bogotá, Colombia. Email: cacusgueng@unal.edu.co \\ ${ }^{3}$ Programa de investigación sobre adquisición y análisis de señales PAAS-UN, Facultad de Ingeniería, Universidad Nacional- \\ Bogotá, Colombia. Email: eamojican@unal.edu.co \\ ${ }^{4}$ Programa de investigación sobre adquisición y análisis de señales PAAS-UN, Facultad de Ingeniería, Universidad Nacional- \\ Bogotá, Colombia. Email: fapavasm@unal.edu.co
}

RECIBIDO: Febrero 9, 2017. ACEPTADO: Mayo 31, 2017. VERSIÓn FINAL: Junio 2, 2017.

\begin{abstract}
RESUMEN
En el presente trabajo se realiza una revisión de algunas de las técnicas de control usadas en microrredes eléctricas, haciendo especial énfasis en las usadas para el modo de operación en modo isla de la microrred, lo cual es una característica esperada en un microrred rural. Por otra parte, se presenta la gestión de despacho como una estrategia de control que impacte en la calidad de energía del sistema de la microrred asociándole concepto de capacidad de atención (Hosting Capacity), ésta última tiene que ver con las restricciones que puede tener el sistema en cuanto a la cargabilidad dada una generación. Es importante mencionar que el trabajo está dentro del marco del proyecto "Incremento en las estrategias sustentables en el uso del recurso de energía eléctrica para la población vulnerable del departamento de Cundinamarca".
\end{abstract}

PALABRAS CLAVE: Microrredes, Generación distribuida, Capacidad de atención, Despacho económico.

\begin{abstract}
In this paper we review control techniques used in electrical microgrids, with a special emphasis on those used for the operation in island mode, which is an expected feature in rural microgrids. On the other hand, the dispatch management is presented as a control strategy that impacts the energy quality of the microgrid associated with the concept of Hosting Capacity, which, in this specific case, it refers to the restrictions the system may have about chargeability given the generation. Finally, it is important to mention that this work was developed in the framework of the project: "Incremento en las estrategias sustentables en el uso del recurso de energía eléctrica para la población vulnerable del departamento de Cundinamarca".
\end{abstract}

KEYWORDS: Microgrids, Distributed generation, Hosting Capacity, Economic Dispatch.

\section{INTRODUCCIÓN}

Entendiendo una microrred de energía eléctrica como un sistema compuesto por generadores y cargas, capaz de autoabastecerse y funcionar de manera autónoma de la red de distribución eléctrica, sistemas en los que se tiene la posibilidad de tener sistemas híbridos que estén constituidos tanto por generación convencional como del uso de fuentes renovables, con el objetivo de asegurar continuidad del servicio como un factor de confiabilidad asociada a la calidad de energía que debe poseer el sistema eléctrico a implementar; por esta razón el estudio de calidad de energía en microrredes ha tomado importancia en los últimos años. Grandes avances se han logrado en el desarrollo de conversores para la generación distribuida (DG) y en menor medida se han desarrollado algoritmos de control que aseguren la calidad de energía. En este artículo se presenta un panorama general del estado actual de las microrredes en cuanto a métodos de control y confiabilidad, así como también se muestra el concepto de Capacidad de atención o Hosting Capacity (HC) y cómo éste puede establecer 
parámetros del sistema útiles en el problema de despacho económico.

La capacidad de atención, entendida este como el máximo de generación distribuida DG (Distributed Generation), penetración para el cual el sistema de potencia opera satisfactoriamente [1]. Más a delante se define con detalle este concepto.

\subsection{Definición y estructura básica de una microrred}

A través del tiempo y atendiendo a los usos y aplicaciones de las microrredes, el concepto y la definición ha cambiado, adaptando la respuesta a cada momento y saber particular. A continuación se mencionan algunas definiciones:

Las microrredes son sistemas de distribución eléctrica que contienen cargas y fuentes de energía distribuidas (tales como generadores distribuidos, dispositivos de almacenamiento, o cargas controlables) que pueden funcionar de manera coordinada y controlada, mientras se conectan a la red o en modo aislado (CIGRÉ C.6.22 Working Group) [2].

El concepto de microrred asume un conjunto de cargas y microfuentes operando como un único sistema controlable que provee energía a su área local. Este concepto presenta un nuevo paradigma en la definición de la operación en la generación distribuida. Para efectos prácticos, la microrred puede ser pensada como una célula controlada del sistema de potencia. Por ejemplo, esta célula puede ser controlada como una única carga que se puede despachar, la cual puede responder en segundos para atender las necesidades del sistema de transmisión. Para el cliente, la microrred puede ser diseñada para atender sus necesidades especiales, tales como: mejorar la confiabilidad local, reducir las pérdidas del alimentador, compatible con voltajes locales, proporcionar una mayor eficiencia a través del uso de residuos de calor, corrección de las caídas de voltaje o proporcionar un suministro ininterrumpido de energía, por nombrar algunas [3].

En la Figura 1 se muestran los elementos observados con mayor frecuencia en una microrred. Dentro de la generación se encuentran fuentes gestionables como las microturbinas, los generadores diésel y las celdas de combustible, y fuentes limitadas como las fuentes de energías renovables, principalmente. También se encuentran baterías como almacenadores de energía que se usan como medio de generación cuando es necesario. Dentro de las cargas se encuentran las cargas críticas que hacen referencia a aquellas que requieren energía ininterrumpida ya que cualquier variación o disturbio eléctrico les afecta significativamente, cargas que son importantes dentro de un proceso en el cual no se puede permitir la falta de funcionamiento, como lo son los dispositivos que se encuentran en un hospital; y las cargas no críticas tales como iluminación, refrigeración calefacción, entre otras según sea el caso. Por último, se presenta la parte de control de la microrred.

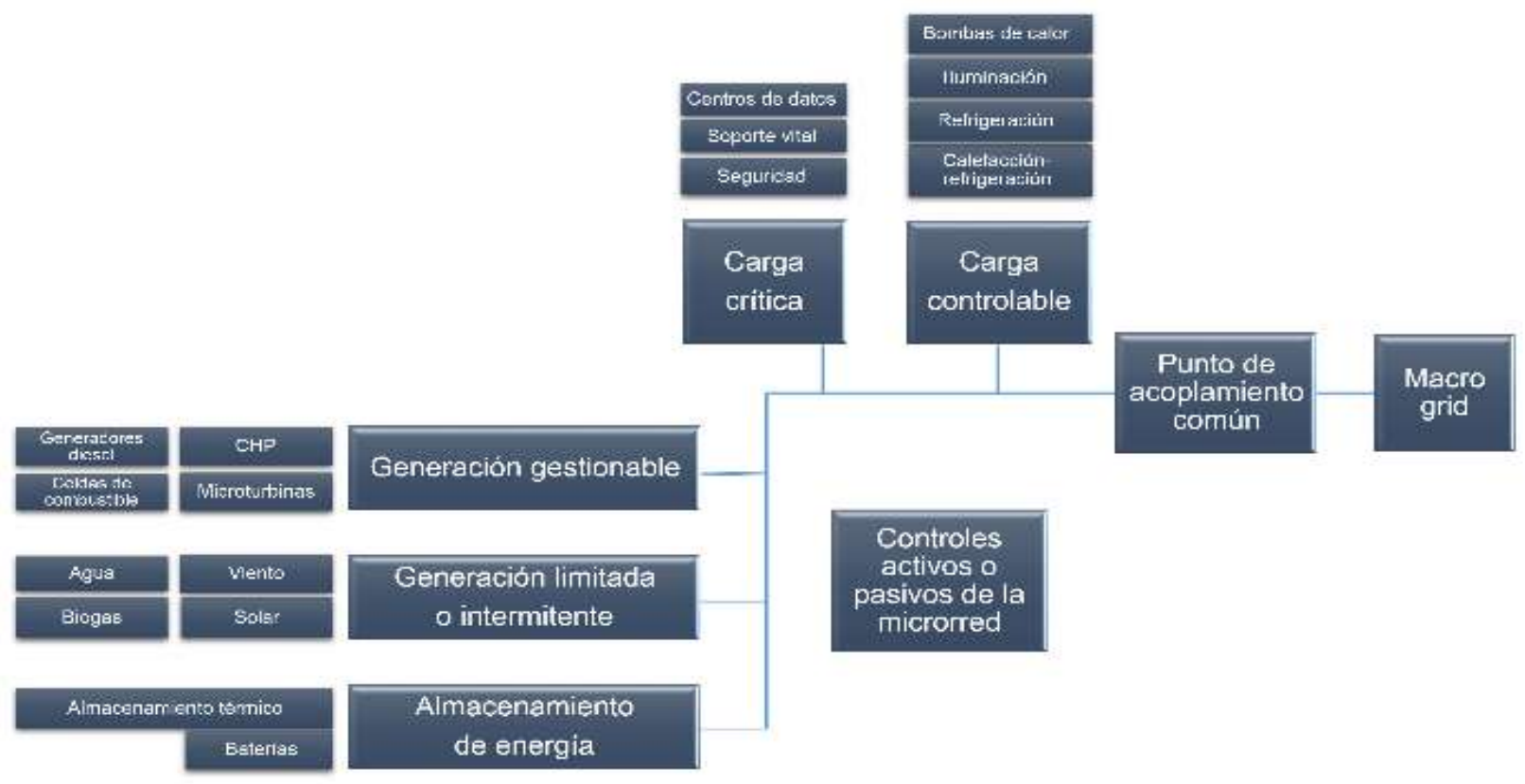

Figura 1. Panorama de los principales componentes de una microrred. Fuente. Elaboración propia. 


\subsection{Panorama actual de microrredes}

Las microrredes son especialmente investigadas en todo el mundo. Por ejemplo, en Japón, proyectos de microrredes son promovidos por varias compañías privadas y por la Organización de Desarrollo de Nueva Energía y Tecnología Industrial (NEDO). Se han llevado a cabo cuatro proyectos de NEDO populares (Aichi Expo, Kyotango, Hachinohe, y Sendai) de 2003 a 2007. NEDO también promueve otros proyectos de microrredes en países asiáticos para investigación en varias condiciones [4]. De estos son China e India donde más interés se tiene en el desarrollo de microrredes debido al mayor crecimiento poblacional de estos países con respecto a otras naciones y por su creciente demanda de energía.

A la fecha no se encuentran aún desarrollos ni investigaciones en microrredes a nivel industrial debido a que los niveles de energía que se manejan no son adecuados para esos propósitos. Algunos ejemplos de microrredes se encuentran en [5], [6], [7].

Ha habido varias iniciativas en Colombia que han abordado el tema de las redes inteligentes, en el que se entienden estas como sistemas que comprenden generación, transmisión, distribución y consumo dentro de un mismo sistema, y en el que cobra vital importancia la red de comunicación del sistema. La primera iniciativa surgió desde la academia con el Proyecto SILICE entre 2006 y 2013, en el que participaron CODENSA, la Universidad de los Andes, la Universidad Nacional de Colombia, la Universidad Industrial de Santander y el CIDET. El propósito del proyecto fue determinar los requerimientos y aproximarse al diseño de microrredes eléctricas en la ciudad de Bogotá, integrando fuentes renovables, estrategias de gestión de demanda, educación en la integración de fuentes alternas, movilidad eléctrica y calidad de energía. En [8], [9] y [10] se muestran algunos de los resultados obtenidos en SILICE.

También surgió una iniciativa en la que participaron algunas empresas del país llamada COLOMBIA INTELIGENTE, descrito en [11], que es un programa nacional de redes inteligentes, que busca promover y desarrollar soluciones al uso eficiente de los recursos energéticos.

A partir de junio de 2015 se inició un proyecto de microrredes en 8 municipios del Departamento de Cundinamarca (Vergara, Jerusalén Guataquí, Quebradanegra, Caparrapí, Nocaima, La Palma y Medina). Este es un proyecto financiado por regalías de la Gobernación de Cundinamarca ejecutado por la Universidad Nacional de Colombia, la Universidad de Los Andes, la Pontificia Universidad Javeriana y por el
Centro Internacional de Física (CIF). El proyecto fue titulado "Incremento en las estrategias sustentables en el uso del recurso de energía eléctrica para la población vulnerable en el departamento de Cundinamarca", se busca con este proyecto llevar suministro de energía eléctrica a partir de energías renovables, principalmente energía fotovoltaica, a las escuelas de los municipios. Como parte de los retos del proyecto anteriormente descrito surge la intención de relacionar el concepto de capacidad de atención, con el problema de despacho económico, para plantear una estrategia que ayude a garantizar la calidad de energía en microrredes rurales.

\subsection{Panorama actual en control y confiabilidad de microrredes}

Las estrategias de control y el comportamiento de la dinámica de la microrred, particularmente en un modo isla, pueden ser notablemente diferentes que las usadas en un sistema convencional de potencia, Las estrategias de control para la generación distribuida (DG), y almacenamiento distribuido de energía eléctrica (DS), por sus siglas en inglés, en una microrred, son seleccionadas según las funciones requeridas y según los posibles escenarios de operación.

Este tipo de control de las unidades DER, que comprenden tanto la generación como el almacenamiento distribuido, son determinados por la naturaleza de las interacciones con el sistema y con otras unidades DER. Las principales funciones de control de las DER son voltaje y frecuencia y/o control de potencia activa y reactiva [12], por ejemplo en [13] se muestran resultados de simulación con mejoras de la regulación de frecuencia utilizando el aumento de inercia de microrredes interconectadas.

Para el registro de parámetros de calidad de energía en microrredes inteligentes, en [14] se plantea una metodología que permite obtener un dispositivo con alta fiabilidad y bajo costo, en términos de producción y operación.

En [15], se presenta una estrategia de gestión de potencia en una microrred conectada, basada en el algoritmo genético para optimización multiobjetivo, la cual tiene como propósito realizar la reducción de los costos operacionales de la microrred y la reducción de la cantidad de emisiones de gases contaminantes.

En [12], se presenta una clasificación de técnicas de control aplicables a microrredes en el que se establece una categorización general de las funciones de control para los DER, en el que se presenta una división entre control de seguimiento de la red y control de formación 
de la red, así como también entre estrategias interactivas y no interactivas.

La categorización mencionada se presenta en la siguiente tabla.

Tabla 1. Clasificación de estrategias de control para acoplamiento de las DER.

\begin{tabular}{|c|c|c|}
\hline & $\begin{array}{c}\text { Controladores } \\
\text { de seguimiento } \\
\text { de la red }\end{array}$ & $\begin{array}{c}\text { Controladores } \\
\text { de formación } \\
\text { de la red }\end{array}$ \\
\hline $\begin{array}{c}\text { Métodos no } \\
\text { interactivos } \\
\text { de Control }\end{array}$ & $\begin{array}{c}\text { Entrega de } \\
\text { potencia (con o } \\
\text { sin MPPT) }\end{array}$ & $\begin{array}{c}\text { Control de } \\
\text { Voltaje y } \\
\text { frecuencia }\end{array}$ \\
\hline $\begin{array}{c}\text { Métodos } \\
\text { interactivos } \\
\text { de Control }\end{array}$ & $\begin{array}{c}\text { Despacho de } \\
\text { potencia real y } \\
\text { potencia reactiva }\end{array}$ & $\begin{array}{c}\text { Repartir carga } \\
\text { (Control droop) }\end{array}$ \\
\hline \multicolumn{2}{|c}{} \\
\hline
\end{tabular}

Fuente. Elaboración propia.

En la estrategia de control de seguimiento de la red, mencionada en la Tabla 1, se simula el comportamiento de una fuente de oscilación en una microrred autónoma. Una unidad de formación de red dentro de la microrred puede ser asignada para regular el voltaje en el punto de acoplamiento común (PCC) y dominar el conjunto de las frecuencias del sistema. La unidad podría ser lo suficientemente grande y tener la suficiente reserva de capacidad para suplir el balance de potencia.

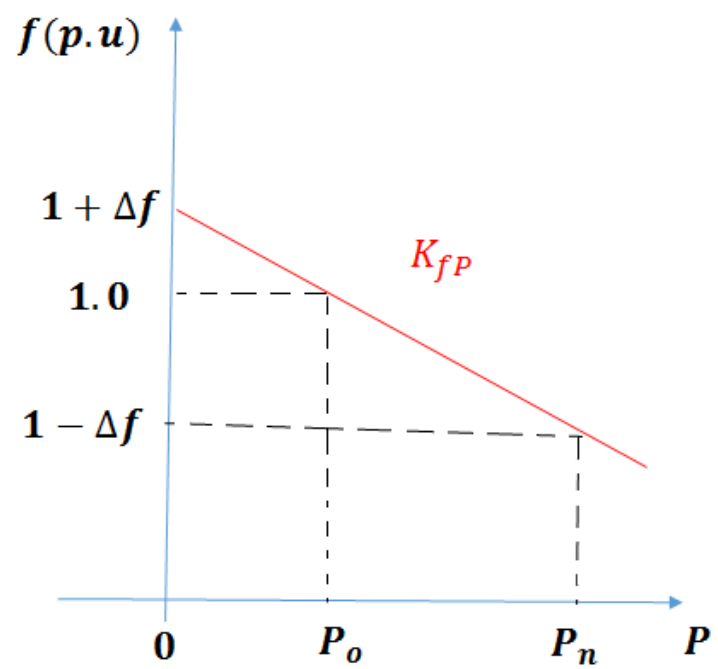

Figura 2. Droop frecuency control, control de potencia activa del sistema. Fuente. Elaboración propia.
Se realiza control de potencia activa moviendo la frecuencia, esto se logra asumiendo una impedancia puramente inductiva, y calculando el coeficiente del control como la máxima desviación de frecuencia (Figura 2) o voltaje (Figura 3) según sea el caso, sobre la potencia máxima activa o reactiva según corresponda.

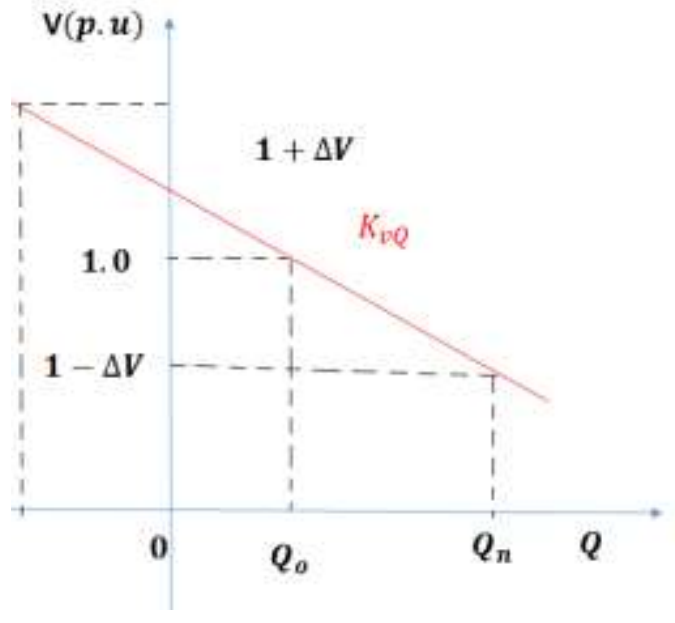

Figura 3. Droop voltage control, control de potencia reactiva del sistema. Fuente. Elaboración propia.

En el control jerárquico, el primario se usa control droop para el momento de conexión a la red, en el secundario para establecer si se va a hacer de manera centralizada o distribuida y el terciario se refiere a gestión de energía dentro del sistema, es allí donde se propone la capacidad de atención como una limitación en el despacho

\subsubsection{Arquitecturas de control.}

Dos arquitecturas comunes de control para microrredes son Control Centralizado y Control Distribuido [16], los cuales hacen parte del control secundario para un sistema eléctrico. Los procedimientos estandarizados e implementaciones sencillas están entre las ventajas del enfoque centralizado. El estudio en [17] presenta el controlador central de una microrred con dos funciones principales de los sistemas de distribución: tener un canal de comunicación con el operador del sistema de distribución y el mercado eléctrico, e intercambio de información con los controladores locales de la microrred y el procesamiento de esa información. En el esquema de control centralizado, el controlador central toma las decisiones sobre el despacho de todas las DGs y sistemas de almacenamiento de energía (ESSs) por su sigla en inglés, de acuerdo con la función objetivo y las restricciones. Es importante tener en cuenta que el reparto de energía y la regulación de tensión son controlados centralmente y los comandos son distribuidos a través de un enlace de comunicación de bajo ancho de banda. Los controladores de alto ancho de banda son distribuidos para cada inversor local. El nivel 
rechazo a perturbaciones logrado por este método es igual al de un solo inversor con un solo controlador de ancho de banda completo. En microrredes donde cada DG tiene su propio controlador y persigue diferentes objetivos, el control distribuido provee aplicabilidad de alta calidad. El número de mensajes transmitidos entre los diferentes dispositivos individuales y el controlador de la microrred, aumenta tanto como aumenta el tamaño de la microrred, necesitando un gran ancho de banda para la comunicación. El control descentralizado puede reducir el número de mensajes y también simplificar la optimización con restricciones especiales reduciéndolo en subproblemas y resolviéndolos localmente [15]. En [16] un sistema diferencial algebraico de control droop descentralizado de potencia activa / frecuencia y potencia reactiva / magnitud de tensión de la microrred es desarrollado y simplificado para determinar fronteras estables de la microrred y diferentes ganancias del control droop.

\subsubsection{Sistemas multiagentes (MAS).}

Una de las aproximaciones para implementar el control distribuido es usando MAS. En esta aproximación cada uno de los elementos controlables de la microrred, tiene agentes asociados a ellos, donde la comunicación y la coordinación de los agentes son gobernadas por la teoría de multiagentes. Los agentes de acoplamiento flexible que forman la masa son física o lógicamente dispersos, y tienen algunas características distintas: 1) sus datos son distribuidos; 2) tienen un proceso de computo asíncrono o simultaneo; 3 ) carecen de información y capacidad de resolver problemas, y 4) ellos interactúan y cooperan el uno al otro, por tanto, su capacidad de resolución de problemas se puede mejorar [20].

\subsubsection{Control cooperativo.}

Para regresar la frecuencia y la tensión a estos valores nominales, se debe emplear un mecanismo de control secundario. El control secundario puede ser control cooperativo, significa que todos los participantes cooperan el uno al otro, y actúan como un solo grupo para alcanzar metas comunes. En [21] se muestra un ejemplo de esto.

\subsubsection{Control adaptativo.}

El control adaptativo es usado para controlar los sistemas con parámetros cambiantes o desconocidos. Como los modos de operación de la microrred pueden cambiar inesperadamente como resultado de perturbaciones en la red de suministro, se proponen modelos de control adaptativo como se ilustran en [16]. Por ejemplo, en [22] se propone una estrategia de control adaptativo para aumentar los controladores existentes y mejorar su rendimiento durante el seguimiento de la respuesta a un dispositivo controlado y modular temporalmente su punto de ajuste de control para lograr un seguimiento cercano al punto de referencia en presencia de perturbaciones.

\subsubsection{Calidad de energía.}

Una de las ventajas más importantes de la microrredes es mejorar la confiabilidad del suministro de los consumidores. Las empresas de electricidad constantemente monitorean los niveles de confiabilidad de los consumidores y realizan las actualizaciones del sistema necesarias para mejorar la disponibilidad del suministro y alcanzar o mantener el rendimiento deseado. La confiabilidad del consumidor es evaluada típicamente en términos del sistema y el promedio de la frecuencia de interrupciones al cliente y/o duración. Causas de interrupción, tales como tormentas, fallos en equipos, etc., impactan los niveles de confiabilidad incrementando la frecuencia promedio y la duración de las interrupciones, sin embargo, cuando se implementa una microrred en su conjunto, estas métricas pueden ser mejoradas significativamente. Esto es debido a la inteligencia intrínseca (control y sistemas de automatización) de las microrredes y al uso de DERs que permiten la operación aislada de la red de suministro. Mejorar la confiabilidad puede ser traducido a beneficios económicos para los consumidores y la empresa de servicios públicos debido a la reducción en costos de interrupciones y energía no suministrada (ENS). La magnitud de estos beneficios depende de la criticidad de carga, el valor de la carga perdida, y la disponibilidad de otras alternativas, tales como la generación de copias de seguridad o viajes de transferencia de carga automática. Los estudios asociados con la confiabilidad de la microrred pueden ser considerados desde dos perspectivas: evaluación y mejora [16]. Relacionado con la evaluación de la microrred, en [23] - [27] se considera este aspecto, en microrredes aisladas con DGs renovables. En [28] y [29] se abordan los efectos de la penetración de DG en la calidad de potencia y la confiabilidad, además de aproximarse al concepto de capacidad de atención.

\section{PLANTEAMIENTO DEL PROBLEMA}

En este capítulo se propone una estrategia que comprende el despacho económico y lo relaciona con el concepto de Capacidad de atención, entendiendo al despacho de energía como una estrategia de control en el nivel terciario y que permite realizar gestión de energía.

El HC permite adicionar una restricción a la ecuación de despecho económico, debido a que, para el sistema, no se puede exceder la cargabilidad del sistema (además de que no se deben sobrepasar los valores límites de frecuencia y tensión), por tanto, se ve acá relacionada la calidad de energía en cuanto a que se buscan medir valores de voltaje y corriente en la línea, para, de esta manera y 
dependiendo de la ubicación de la generación distribuida, se puedan establecer parámetros de capacidad de atención tanto de manera local cómo global, según las condiciones del sistema. Se presentan por tanto las definiciones en la forma que permitirían abordar el problema de forma que se puedan relacionar los conceptos.

Adicionalmente, y para ilustrar esto, se ilustra un pequeño sistema que busca emular la disposición de 3 generadores distribuidos donde se evalúa la capacidad de atención que ayuda a establecer límites en cuanto a que la generación que se tenga no comprometa la cargabilidad del sistema. Teniendo en cuenta tales restricciones es como se evaluaría el despacho, que se entiende como sigue:

Maximizar la utilidad de todos los generadores sujetos al balance de potencia y capacidad de generación es el principal objetivo del problema de despacho económico (EDP). Un concepto similar de despacho puede ser usado en microrredes para coordinar las cargas eléctricas y la capacidad de generación de los generadores distribuidos.

Para el estudio se puede asumir que cada generador distribuido es conectado a un bus por medio de un inversor de voltaje de fuente [30]. El control de bajo nivel de los inversores está basado en un controlador de ganancia droop propuesto en [31] para control en generaciones distribuidas. Se considera una configuración de red con pérdidas de potencia en los alimentadores e integración de energías renovables distribuidas. El problema de despacho económico bajo estas consideraciones, se formula como sigue.

$$
\max J(p)=\sum_{i=1}^{N} J_{i}\left(P_{i}\right)+\sum_{k=1}^{K} J_{k}\left(P_{\omega k}\right)
$$

s.t

$$
\begin{aligned}
& \sum_{i=1}^{N} P_{i}+\sum_{k=1}^{k} P_{\omega k}=\sum_{j=1}^{M} P_{l j}+L\left(P_{i}\right)=P_{l} \\
& 0 \leq p_{i} \leq P_{\text {max }_{i}} \forall i=1,2, \ldots \ldots, N, \\
& 0 \leq p_{\omega k} \leq P \omega_{\text {max }_{i}} \forall k=1,2, \ldots \ldots, K,
\end{aligned}
$$

Donde $p_{i}$ es la potencia suministrada por cada $D G_{i}$ convencional; $N$ es el número de generaciones distribuidas; $K$ es el número de generaciones distribuidas renovables; $P_{\omega k}$ es la potencia nominal para el $k-$ ésimo generador distribuido renovable; $P_{l}$ es la potencia total demandada por la microrred, incluyendo pérdidas; $M$ es el número de cargas; $P_{l j}$ es la carga demandada del nodo $j ; L\left(P_{i}\right)$ es la pérdida de potencia en la microrred;
$P_{\text {max }_{i}}$ es la capacidad de generación nominal del $i-$ ésimo $D G ; P \omega_{\text {max }_{i}}$ es la máxima capacidad de generación del $k$-ésimo generador distribuido renovable; $J_{i}\left(P_{i}\right)$ es la función de cada $D G$ tradicional y, $J_{k}\left(P_{\omega k}\right)$ es la función de utilidad de cada fuente de energía distribuida renovable.

El criterio de despacho económico, es un concepto fundamental en la solución del EDP. Éste establece que todos los DGs deben funcionar a las mismas utilidades marginales. Estas son definidas como la primera derivada de las funciones de utilidad corriendo al precio de oferta. De forma general esto es

$$
\begin{aligned}
& \frac{d J_{1}}{d p_{1}}\left(\frac{1}{1-\frac{\partial L}{\partial p_{1}}}\right)=\cdots \frac{d J_{r}}{d p_{r} N}\left(\frac{1}{1-\frac{\partial L}{\partial p_{r} N}}\right) \\
& \frac{d J_{1}}{d p_{w} 1}\left(\frac{1}{1-\frac{\partial L}{\partial p_{1}}}\right)=\cdots=\frac{d J_{k}}{d p_{w} K}\left(\frac{1}{1-\frac{\partial L}{\partial p_{w} K}}\right)=\mu,
\end{aligned}
$$

Para algún $\mu>0$ tal que $\sum_{i=1}^{N} P_{i}+\sum_{k=1}^{K} P_{\omega k}=P_{l}$, donde $\frac{\partial L}{\partial p}$ es la pérdida que tiene gradualmente un generador, $\mathrm{y}$ $\left(\frac{1}{1-\frac{\partial L}{\partial p}}\right)$ es el factor de penalidad por pérdida de potencia.

\subsection{Problema de despacho económico}

En este caso se utiliza un algoritmo jerárquico tradicional. Este algoritmo es basado en algoritmos de planificación económica presentados para el nivel alto, un control integral-proporcional tradicional para el control de carga secundario y en el nivel bajo un control droop desacoplado. La arquitectura jerárquica tradicional tiene los tres niveles en diferentes escalas de tiempo. Para el nivel alto, un algoritmo tradicional de optimización puede ser usado tal como descomposición dual o método de punto interior. La descomposición dual presenta baja velocidad de convergencia y es computacionalmente costosa, lo cual crea un factor limitante en la implementación práctica. En general, esos métodos centralizados convencionales requieren que la función de costo sea convexa.

Las funciones de costo convexas han sido ampliamente usadas para análisis preliminares pero en casos prácticos las funciones de costo son no-convexas. Tales casos prácticos pueden considerar múltiples opciones de combustible y efectos en el punto de evaluación de la carga resultando en problemas de despacho económico no-convexos. 
Normalmente se resuelve el problema de despacho económico con una S-function de MATLAB de optimización estática. Este algoritmo puede ser descrito matemáticamente como un problema de minimización de costo con las mismas restricciones que se muestran en (1) y la función de costo de cada generador se asume, por simplicidad, como una función cuadrática como sigue:

$L_{i}\left(p_{i}\right)=a_{i}+b_{i} p_{i}+c_{i} p_{i}^{2}$

Los parámetros variables en el tiempo pueden ser costos y variaciones en la carga. Sin embargo, por simplicidad, se pueden presentar constantes.

Definido esto se introduce el concepto de capacidad de atención ya que, si se interpreta el problema de despacho económico bajo esa idea el valor de $P_{\max _{i}}$ se está considerando fijo. Al introducir la idea de capacidad de atención, para la metodología que se propone, se está haciendo que este valor de $P_{\max _{i}}$ ya no sea un valor fijo al ser dependiente de voltaje. En la sección siguiente se define el concepto de Capacidad de atención y se involucra en el entorno de despacho económico.

\subsection{Capacidad de atención (Hosting Capacity) en problemas de despacho económico}

La capacidad de atención es definida como la cantidad de generación distribuida para la cual el desempeño del sistema se vuelve inaceptable, en otras palabras, es la máxima capacidad de generación que puede ser conectada sin resultar en una calidad o confiabilidad inaceptable para otros consumidores [28], [29].

En la práctica, muchos de los índices de desempeño son definidos de forma que un bajo valor en la generación, corresponde a un mejor desempeño. El deterioro se desarrolla al incrementar la cantidad de generación distribuida lo que corresponde a elevar el nivel del índice de desempeño tal como se muestra en la Figura 4.

Hay varias opciones para definir los índices de desempeño, en Figura 5 se muestra cómo el índice de desempeño decrece con el incremento de generación distribuida.

Un ejemplo de esto es la máxima magnitud de tensión experimentada por alguno de los consumidores en una red de distribución.

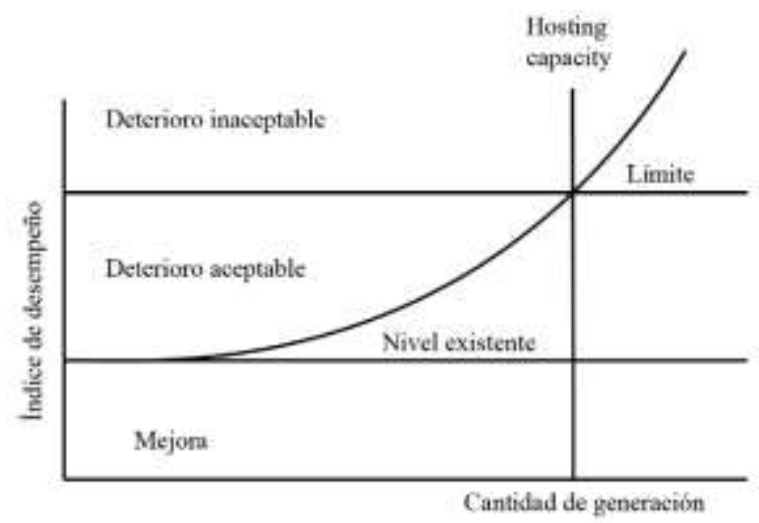

Figura 4. Capacidad de atención cuando al aumentar la cantidad de generación aumenta el índice de desempeño. Fuente. Elaboración propia.

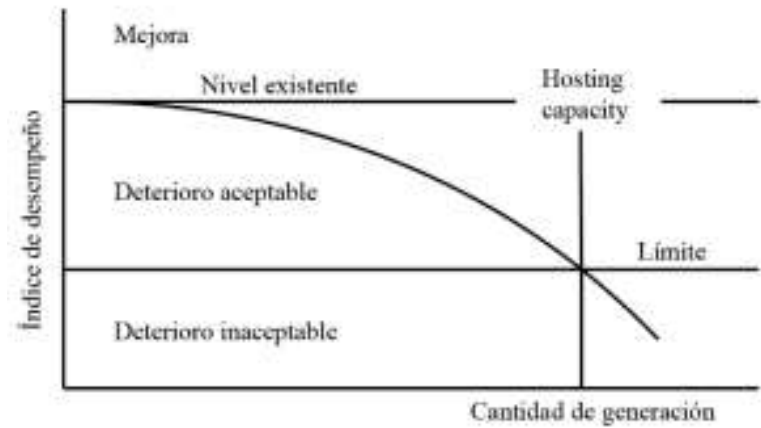

Figura 5. Capacidad de atención cuando al aumentar la cantidad de generación disminuye el índice de desempeño. Fuente. Elaboración propia.

Existen también casos en los cuales la introducción de generación distribuida inicialmente resultará en un incremento del desempeño del sistema de energía. Pero, para una gran cantidad de generación distribuida el comportamiento se deteriora. Este hecho se muestra en la Figura 6.

Dos capacidades de atención se pueden distinguir en este caso: La primera capacidad de atención arriba de la cual el desempeño del sistema se deteriora comparado con el caso de no-generación, y la segunda capacidad de atención arriba de la cual el desempeño del sistema se vuelve inaceptable. Ejemplos de esto son el riesgo de sobrecarga y las pérdidas en la red. Ambos inicialmente se reducen, pero se incrementan cuando un gran número de generaciones distribuidas están conectadas. 


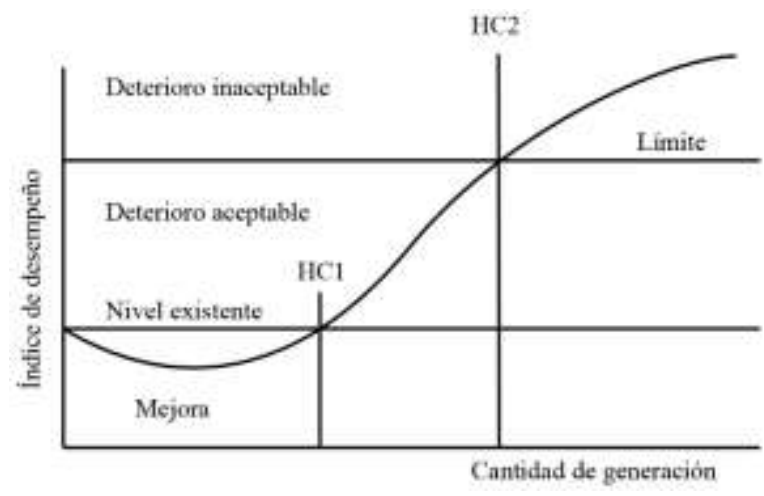

Figura 6. Capacidad de atención en el caso del índice de desempeño. Fuente. Elaboración propia.

La primera capacidad de atención es frecuentemente referida de manera implícita en los debates sobre la confiabilidad. La conexión de generación distribuida debería, de acuerdo a algunos, no resultar en un deterioro en la confiabilidad del suministro. Aparte del error de que la confiabilidad es cuantificada por un número de índices, el error hecho en tal afirmación es que un cierto deterioro del suministro debería ser aceptable para la mayoría de los consumidores.

De forma simplificada el método de la capacidad de atención, es como sigue:

- Escoger un fenómeno y uno o más índices de desempeño.

- Determinar un límite o límites adecuados.

- Calcular el índice de desempeño como una función de la cantidad de generación.

- Obtener la capacidad de atención.

Para saber cuánta generación distribuida puede ser conectada, es importante definir indicadores de desempeño adecuados. La capacidad de atención está basada en eso. La escogencia de índice y límite tendrá una gran influencia en la cantidad de generación distribuida que puede ser aceptada.

Esta aproximación da una capacidad de atención para cada fenómeno (magnitud de voltaje, sobrecarga, etc.) o incluso para cada índice (número de interrupciones, duración de las interrupciones, etc.). Es el más bajo de esos valores el que determina cuánta generación distribuida puede ser conectada antes de que se requieran cambios en el sistema.

La conexión de un generador a la red de distribución resultará en una subida de voltaje en las terminales del generador. El aumento relativo de voltaje, como se describe en [23], es aproximadamente igual a

$\frac{\Delta V}{V}=\frac{R \times P_{g e n}}{V^{2}}$
Donde $R$ es la resistencia de fuente en los terminales del generador, $P_{g e n}$ es la potencia activa inyectada y $V$ el voltaje nominal. Esta aproximación es válida para todos los casos prácticos a nivel de distribución.

La capacidad de atención es calculada de (4) como

$$
P_{\max }=\frac{V^{2}}{R} \times \delta_{\max }
$$

Donde $\delta_{\text {max }}=\frac{\Delta V_{\text {max }}}{V}$ es el margen de voltaje relativo (en porcentaje) y $\Delta V_{\max }$ es el margen de voltaje absoluto (en voltios).

De (5) se puede ver que la capacidad de atención es más pequeña para un margen pequeño de sobretensión $\delta_{\max }$ y para una distancia grande de sobretensión principal (mayor $R$ ). Ambos parámetros varían a lo largo de la línea y no es posible dar reglas generales para cuál ubicación determina la capacidad de atención.

Como ejemplo, el aumento máximo de tensión admisible, con conexión de un generador distribuido, es ese que lleva la máxima magnitud de voltaje exactamente al límite de sobretensión. El "margen de sobretensión" es definido entonces como la diferencia entre la máxima magnitud de voltaje para un consumidor dado y el límite de sobretensión.

\section{RESULTADOS}

Se utiliza para el ejemplo un sistema con tres generadores y dos cargas como se ilustra en la Figura 7, una de las cuales se deja fija mientras que la otra cambia a los $0.35 \mathrm{~s}$. Esta configuración es propuesta para ver los efectos del planteamiento que se hace en el documento. Es decir, se quiere ver que, por un lado, se lleve a cabo el despacho de forma que el sistema supla eficientemente la demanda de carga cuando hay cambios y, por otro lado, ver el efecto de agregar una generación distribuida adicional con valores de potencia para que estén antes y después de superar la capacidad de atención.

Para efectos de simulación y análisis del ejercicio que se plantea se utilizan los datos mostrados en la Tabla 2.

Tabla 2. Valores de referencia

\begin{tabular}{|c|c|}
\hline \multicolumn{2}{|c|}{ Valores de referencia } \\
\hline Medida & Valor \\
\hline L1 & $12000 \mathrm{~W}$ \\
\hline L2 & $2000-15000 \mathrm{~W}$ \\
\hline Rlínea & $0.23 \Omega$ \\
\hline Vlínea & $300 \mathrm{~V}$ \\
\hline
\end{tabular}

Fuente. Elaboración propia. 
Al tener estas características de línea, tomando como indicador el voltaje y dando a este un límite de 1\%, la capacidad de atención será de $4000 W$. Debido a que un cambio de un $1 \%$ en la variación es poco notable en la simulación que se tiene del sistema, y sólo para efectos de simulación -ya que evidentemente en la vida real este margen es totalmente inaceptable-, al dar un valor límite de $10 \%$, el valor de la capacidad de atención será de $40000 \mathrm{~W}$. Luego de este valor el estado del sistema pasa a ser inaceptable.

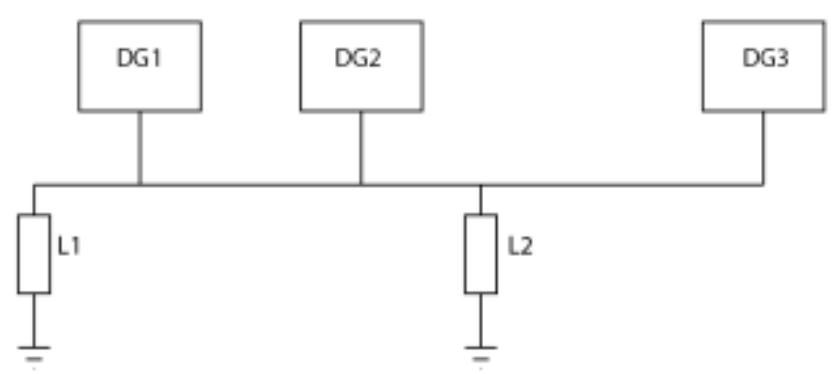

Figura 7. Sistema de prueba. Fuente. Elaboración propia.
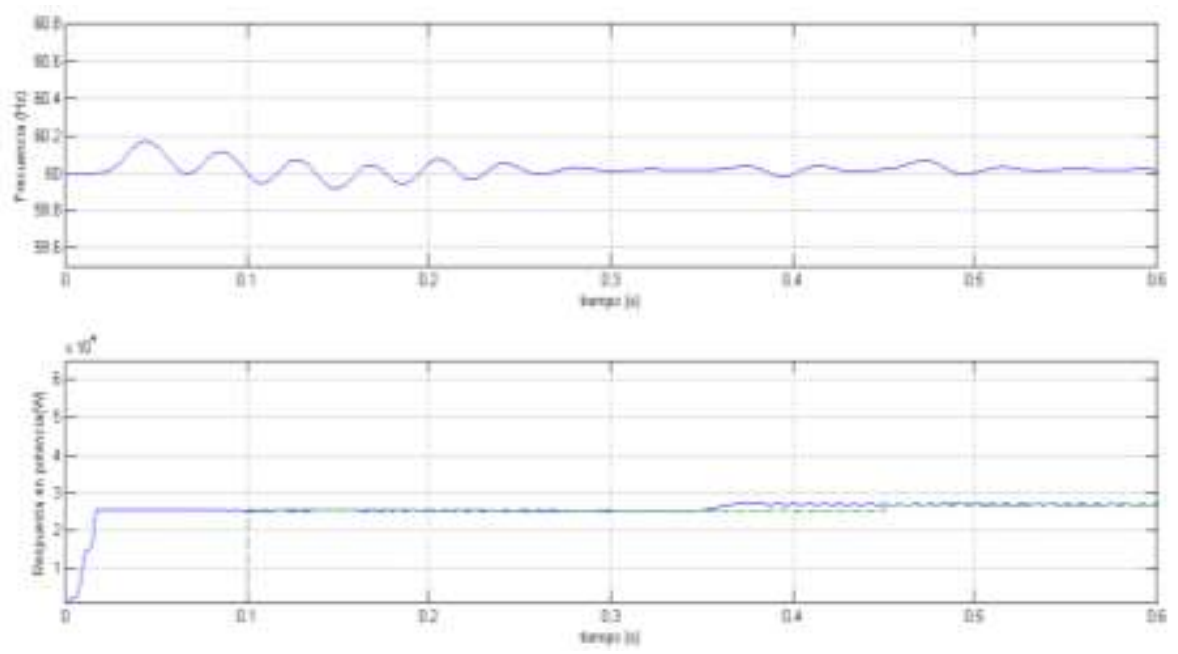

Figura 8. Respuesta del sistema en frecuencia y potencia con solución de despacho económico. Fuente. Elaboración propia.

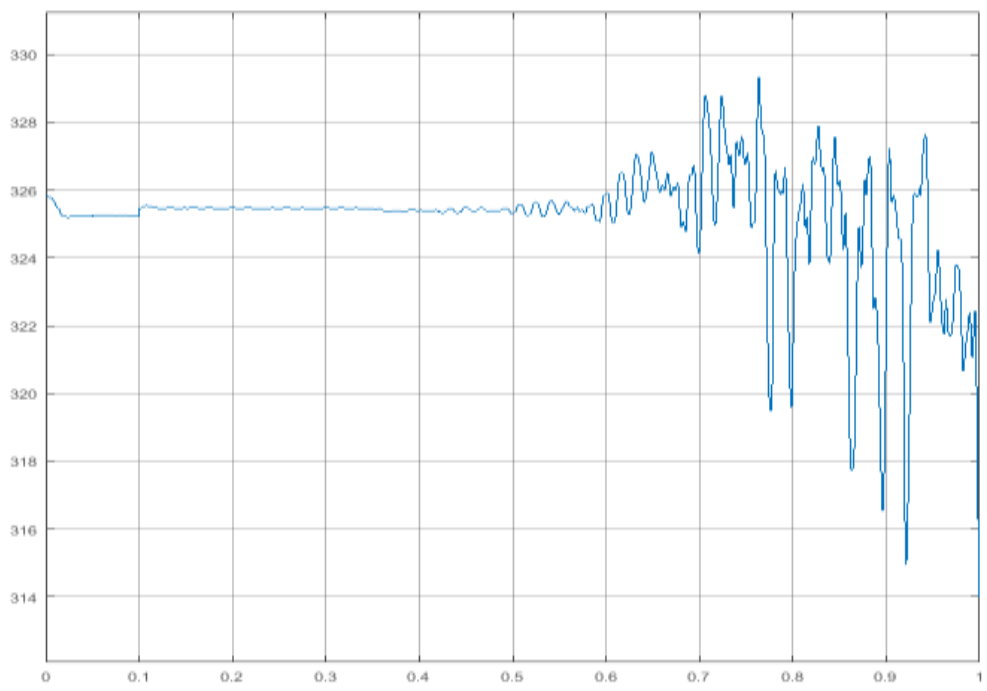

Figura 9. Respuesta del sistema en magnitud de tensión en el nodo de carga variable con solución de despacho económico cuando se supera la capacidad de atención. Fuente. Elaboración propia. 
Inicialmente se tiene el sistema para probar que el problema de despacho económico con los parámetros con que se plantea, funcione de manera óptima en cuanto a suplir la demanda de carga, así, cuando hay una fuerte variación de carga esta debe ser atendida por la generación debido a que así lo estable el algoritmo de generación, los resultados se muestran en la Figura 8.

Acá se pueden observar dos cosas: por un lado, que el sistema de control mantiene al sistema dentro del rango de frecuencia y, por otro lado, que la condición de que la potencia demandada por el sistema sea igual a la potencia generada se cumple. Puede observarse, en la parte inferior de la figura, que el sistema responde a los cambios en la demanda, realizando el despacho y cumpliendo con lo establecido en cuanto a condiciones de suplir con la demanda.

Ahora se introduce en el sistema un generador adicional en el nodo de la carga 2 ( $L 2)$ para ver el comportamiento cuando la potencia de este generador que se incluye aumenta gradualmente de forma que supera la capacidad de atención. Para este generador adicional, cada $0.1 \mathrm{~s}$ se aumenta la potencia en pasos como sigue:

$[200,2000,10000,20000,40000,50000] W$, lo que indica que a los $0.5 \mathrm{~s}$ se alcanza la capacidad de atención, y que a partir de este valor el índice de desempeño estudiado queda fuera de los límites aceptables, lo que debería generar cambios en el comportamiento del sistema. Los resultados se muestran a continuación en la Figura 10.

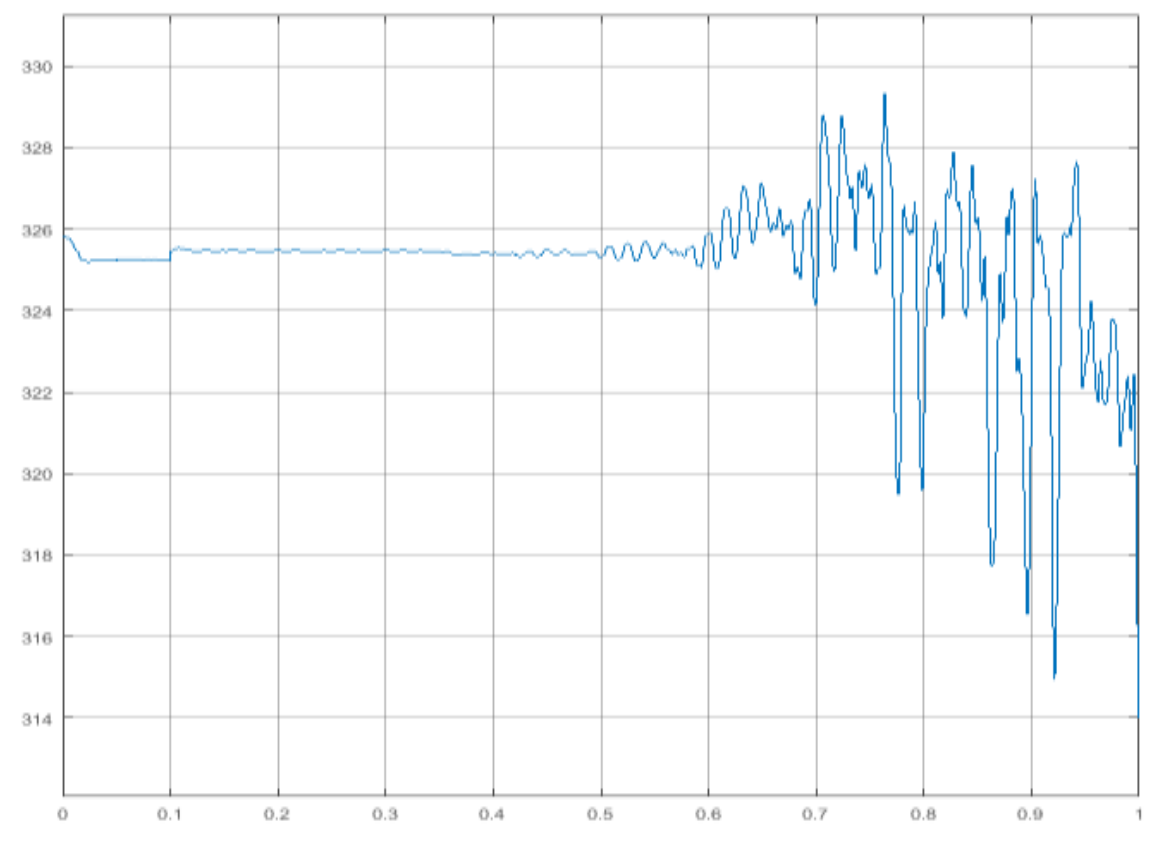

Figura 10. Respuesta del sistema en magnitud de tensión en el nodo de carga variable con solución de despacho económico cuando se supera la capacidad de atención. Fuente. Elaboración propia.

Acá se observan cambios altamente notorios en el comportamiento del sistema. Al observar la frecuencia y comparar los resultados con los de la Figura 6, se observa que el despacho se cumple eficientemente para suplir la carga que se demande en el sistema, pero que a medida que se aproxima y se supera el límite en la capacidad de atención, se obtienen resultados no deseados.

Se había hecho mención de que pasado el tiempo en que se supera la capacidad de atención del sistema, este tendría sus parámetros en límites inaceptables donde se comprometía directamente la estabilidad del sistema, de forma particular el índice que se observe tendría comportamientos y valores no aceptables comportamiento observado en la figura 7.

Se observa el esfuerzo del sistema por mantener el valor de tensión estable a través del despacho, pero una vez se ha superado el límite de capacidad de atención del sistema ya no es posible mantener la estabilidad. No hay que olvidar que el límite en capacidad de atención no sólo está relacionado a los cambios que se pueden observar en la magnitud de la tensión, como se ilustra en el ejemplo, sino que también está involucrado con los otros índices de desempeño del sistema como lo son la frecuencia y la cargabilidad y los demás mencionados en la sección 2 , por tanto si la frecuencia llega a un punto más allá de los límites de estabilidad del sistema, es de esperarse que 
más allá del límite en capacidad de atención de este indicador el sistema no pueda recuperarse ni permanecer dentro de límites estables.

\section{DISCUSIÓN Y TRABAJO FUTURO}

En este trabajo se plantea la integración del concepto de Capacidad de atención dentro del algoritmo de despacho óptimo usando técnicas de optimización. Se analiza un circuito sencillo donde el concepto de capacidad de atención se implementa para el problema de despacho con generadores distribuidos.

Es necesario, y es lo queda de ejercicio a realizar, la modificación en las restricciones de la formulación del problema de despacho económico a optimizar de tal manera que se tenga involucrada la restricción que se tiene por la capacidad de atención del sistema, de tal manera que el despacho pueda realizarse de forma que al llegar al límite de algún índice de desempeño, el despacho se haga de forma que no comprometa la estabilidad del sistema. Esto implica por tanto la modificación de la función a optimizar en cuanto a que los coeficientes se deben ver modificados de forma que incluyan las características de las generaciones distribuidas que se puedan integrar al sistema.

El despacho responde a las demandas del sistema y la inclusión de generaciones distribuidas muestra que el comportamiento del sistema se sale de los parámetros impuestos cuando se supera la capacidad de atención de algún indicador de desempeño del sistema. Lo que se espera es que bajo otras condiciones de control, se pueda observar con mucho más detalle, cómo para algunos índices de desempeño, sobrepasar la capacidad de atención lleva a estos índices a valores no deseados. Es decir que una vez solucionadas la inclusión de la generación distribuida dentro de las restricciones y la optimización del sistema, se puedan observar otros índices de desempeño para asegurar con mayor criterio la estabilidad del sistema. Este análisis permitirá el buen diseño del sistema de distribución lo que tendrá como consecuencia asegurar de forma adecuada la calidad de energía del sistema.

\section{REFERENCIAS}

[1] M.H.J. Bollen, M. Häger. "Power quality: interactions between distributed energy resources, the grid, and other customers," Electric Power Quality Utilization Magazine, Vol.1, No 1, Jul - Sep. 2004.

[2] J. Romankiewicz, M. Qu, C. Marnay, and N. Zhou, "International micro-grid assessment: Governance, incentives, and experience (IMAGINE)," pp. 1-21, 2013.
[3] R. H. Lasseter, "Microgrids," Power Eng. Soc. Winter Meet., vol. 1, pp. 146-149, 2001.

[4] H. Kakigano, Y. Miura, and T. Ise, "Lowvoltage bipolar-type DC microgrid for super high quality distribution," Power Electron. IEEE, vol. 25, no. 12, pp. 3066-3075, 2010.

[5] R. V. Sánchez, H. G. S. Uruchurtu, J. L. S. Farías, G. Vidrio, and R. Nieva, "Introducción al concepto de microrredes," pp. 102-108. 2005.

[6] M. Barnes, J. Kondoh, H. Asano, J. Oyazarbal, R. H. Lasseter, G. Van-takaramanan, N. Hatziargyrio, and T. Green, "Real-world microgrids- An overview," IEEE Power energy mag., vol. 6, no. 3, pp. 26-94, 2008.

[7] M. Häshmi, S. Hänninen, and K. Mäki, "Survey of Smart Grid Concepts, Architectures, and Technological Demonstrations Worldwide," pp. 1-7, 2011.

[8] M. Ortiz, W. Gómez, G. Archila, G. Ordoñez, J. Petit. "Topological and Technological Characterization of a Residential Energy Management System in the Smart Grid Context", VII Simposio Internacional sobre Calidad de Energía - SICEL 2013. Medellín, Colombia, Nov 27-29, 2013.

[9] A. Pavas, L.E. Luna, C. Laiton, H. C. Torres, M. Romero, L. Gallego, E. Mojica, H. Torres, "Towards a smart city - SILICE III: Microgrids and electric vehicles analysis", VII Simposio Internacional sobre Calidad de Energía - SICEL 2013. Medellín, Colombia, Nov 27-29, 2013.

[10] A. M. Ospina, et al. "Analysis and Design of a Microgrid for a Codensa's Test Circuit", VII Simposio Internacional sobre Calidad de Energía - SICEL 2013. Medellín, Colombia, Nov 27-29, 2013.

[11] R. Cespedes, A. Aldana, E. Parra, R. Lopez, M. E. Ruiz, "Implementation of Smart Grids in the Colombian Electrical Sector”, IEEE ISGT 2011, Oct.19-21, 2012.

[12] F. Katiraei, R. Iravani, N. Hatziargyriou and A. Dimeas, "Microgrids Management - Control and Operation Aspects of Microgrids", IEEE Power and Energy Magazine, vol. 6, iss. 3, May - June 2008, pp. 5465.

[13] Toro Tovar, Billy Vladimir; Mojica Nava, Eduardo; Rivera, Sergio. Mejoras de la Regulación de Frecuencia Utilizando el Aumento de Inercia de Microrredes Interconectadas. Revista UIS Ingenierías, S.1., v. 16, n. 2, jul. 2017. 
[14] Zuñiga Cortes, Fabian Andres; Caicedo Bravo, Eduardo Francisco; LÓPEZ SANTIAGO, Danny Mauricio. Gestión Óptima de la Potencia Eléctrica en una Microgrid Conectada, basada en el Algoritmo Genético para Optimización Multiobjetivo Moga. Revista UIS Ingenierías, [S.1.], v. 15, n. 2, nov. 2016.

[15] Perdomo Orjuela, Luis Eduardo; Alfonso Rodríguez, Andrés; Santamaría, Francisco. Metodología para el registro de parámetros de calidad de energía en microrredes Inteligentes. Revista UIS Ingenierías, [S.1.], v. 15, n. 2, Nov. 2016.

[16] B. Starfield, "State of the art in research on Microgrids: A review," J. Health Polit. Policy Law, vol. 31, no. 1, pp. 11-32, 2006.

[17] F. Dorfler, J. Simpson-Porco, and F. Bullo, "Breaking the Hierarchy: Distributed Control and Economic Optimality in Microgrids," IEEE Trans. Control Netw. Syst., vol. 5870, no. c, pp. 1-1, 2015.

[18] L. K. Gan, D. E. Macpherson, and J. K. H. Shek, "Synchronisation control and operation of microgrids for rural/island applications," in Proceedings of the Universities Power Engineering Conference, Dublin, Ireland, Sep, 2013.

[19] S. V. Iyer, M. N. Belur, and M. C. Chandorkar, “A generalized computational method to determine stability of a multi-inverter microgrid," IEEE Trans. Power Electron., vol. 25, no. 9, pp. 2420-2432, 2010.

[20] G. Zheng and N. Li, "Multi-Agent Based Control System for Multi-Microgrids," 2010 Int. Conf. Comput. Intell. Softw. Eng., no. 1, pp. 1-4, 2010.

[21] A. Bidram, A. Davoudi, F. L. Lewis, and J. M. Guerrero, "Distributed cooperative secondary control of microgrids using feedback linearization," IEEE Trans. Power Syst., vol. 28, no. 3, pp. 3462-3470, 2013.

[22] A. Mehrizi-Sani and R. Iravani, "Online set point modulation to enhance microgrid dynamic response: Theoretical foundation," IEEE Trans. Power Syst., vol. 27, no. 4, pp. 2167-2174, 2012.
[23] H. Daneshi and H. Khorashadi-Zadeh, "Microgrid energy management system: A study of reliability and economic issues," 2012 IEEE Power Energy Soc. Gen. Meet., pp. 1-5, 2012.

[24] B. Falahati, A. Kargarian, and Y. Fu, "Timeframe capacity factor reliability model for isolated microgrids with renewable energy resources," in IEEE Power and Energy Society General Meeting, 2012.

[25] S. Kennedy, "Reliability evaluation of islanded microgrids with stochastic distributed generation," in 2009 IEEE Power and Energy Society General Meeting, PES '09, 2009.

[26] A. K. Basu, S. Chowdhury, and S. P. Chowdhury, "Distributed energy resource capacity adequacy assessment for PQR enhancement of CHP micro-grid," in IEEE PES General Meeting, PES 2010, 2010.

[27] R. Yokoyama, T. Niimura, and N. Saito, "Modeling and evaluation of supply reliability of microgrids including PV and wind power," in IEEE Power and Energy Society 2008 General Meeting: Conversion and Delivery of Electrical Energy in the 21st Century, PES, 2008.

[28] M. Bollen, F. Hassan, "Power System Performance," en Integration of Distributed Generation in the Power System, 1 ed. Wiley-IEEE press, 2011, cap. 3 , sec. 3.3 , pp. $88-91$.

[29] Vanessa Quintero-Molina, Daniel Vega, Uriel Mora, Andrés Pavas. "Impact assessment of distributed generation on the power quality and reliability of distribution grid". Power Electronics and Power Quality Applications (PEPQA), 2015 IEEE Workshop on. Bogotá, Colombia, Jun, 2015. pp.1-7.

[30] J. Lopes, C. Moreira, and A. Madureira, "Defining control strategies for microgrids islanded operation", IEEE Transactions on Power Systems, 2006, 21, (2), pp. 916-924.

[31] Y. Li, and Y. Li, "Power management of inverter interfaced autonomous microgrid based on virtual frequency-voltage frame", IEEE Transactions on Smart Grid, 2011, 2, (1), pp. 30-40. 